\title{
Successful Excision of a Cystic Tumor of the Atrioventricular Nodal Region
}

\author{
Shunsuke Saito, MD; Junjiro Kobayashi, MD; Osamu Tagusari, MD; Ko Bando, MD; \\ Kazuo Niwaya, MD; Hiroyuki Nakajima, MD; Masakazu Yamagishi, MD*; \\ Toshikatsu Yagihara, MD; Soichiro Kitamura, MD
}

\begin{abstract}
Cystic tumor of the atrioventricular nodal region is a rare cardiac primary tumor that can cause heart blockage and sudden death. Antemortem diagnosis and successful excision of the atrioventricular nodal region are extremely rare. A 45-year-old woman who presented with palpitations is reported. Electrocardiography revealed firstdegree atrioventricular block. Echocardiography, computed tomography, and magnetic resonance imaging scans revealed a cystic mass attached to the interatrial septum. Complete surgical excision of the mass was achieved, although placement of a permanent pacemaker was required for complete heart blockage. Histopathological examination revealed the mass to be a cystic tumor of the atrioventricular nodal region. A 5-year follow-up has revealed no sign of recurrence. (Circ J 2005; 69: 1293-1294)
\end{abstract}

Key Words: Atrioventricular node; Cystic tumor; Heart block; Pacemaker

C ystic tumor of the atrioventricular nodal region is a rare primary cardiac tumor. It can cause various degrees of heart blockage, and is the smallest tumor capable of causing sudden death!-3 Although there have been approximately 70 reported cases of atrioventricular nodal region in published reports to date, most were diagnosed postmortem. Antemortem diagnosis and successful excision of this type of tumor are extremely rare. Here we report a case of cystic tumor of the atrioventricular nodal region in which the tumor was detected preoperatively and successfully excised.

\section{Case Report}

A 45-year-old woman who presented with dyspnea on effort and palpitations visited her primary care physician. A resting electrcardiogram (ECG) revealed sinus rhythm and first-degree atrioventricular block. Sporadic ventricular paroxysmal contraction was found on Holter ECG. Because echocardiography showed an intracardiac tumor, she was referred to our institute for surgical evaluation. Transesophageal echocardiography and computed tomography (Fig 1A) revealed a $28-27 \mathrm{~mm}$ circular tumor with no stalk on the interatrial septum (IAS)4 On magnetic resonance imaging (MRI), the tumor was of high intensity on T1weighted images and isointense with myocardium on $\mathrm{T}_{2}$ weighted images (Fig 1B).

She underwent surgery to resect the tumor. Under a median sternotomy and standard cardiopulmonary bypass with warm blood cardioplegia, the right atrium was opened, to reveal a $30 \mathrm{~mm}$ round cyst attached to the IAS in the area of the triangle of Koch. The cyst was incised

(Received March 3, 2005; revised manuscript received June 27, 2005; accepted July 19, 2005)

Departments of Cardiovascular Surgery and *Cardiology, National Cardiovascular Center, Suita, Japan

Mailing address: Osamu Tagusari, Department of Cardiovascular Surgery, National Cardiovascular Center, 5-7-1 Fujishirodai, Suita 575-8575, Japan. E-mail: otagusar@hsp.ncvc.go.jp and yellow caseous material was found within it. Rapid cytodiagnosis was done and revealed neither malignant cells nor bacteria in the fluid. The IAS at the portion of the
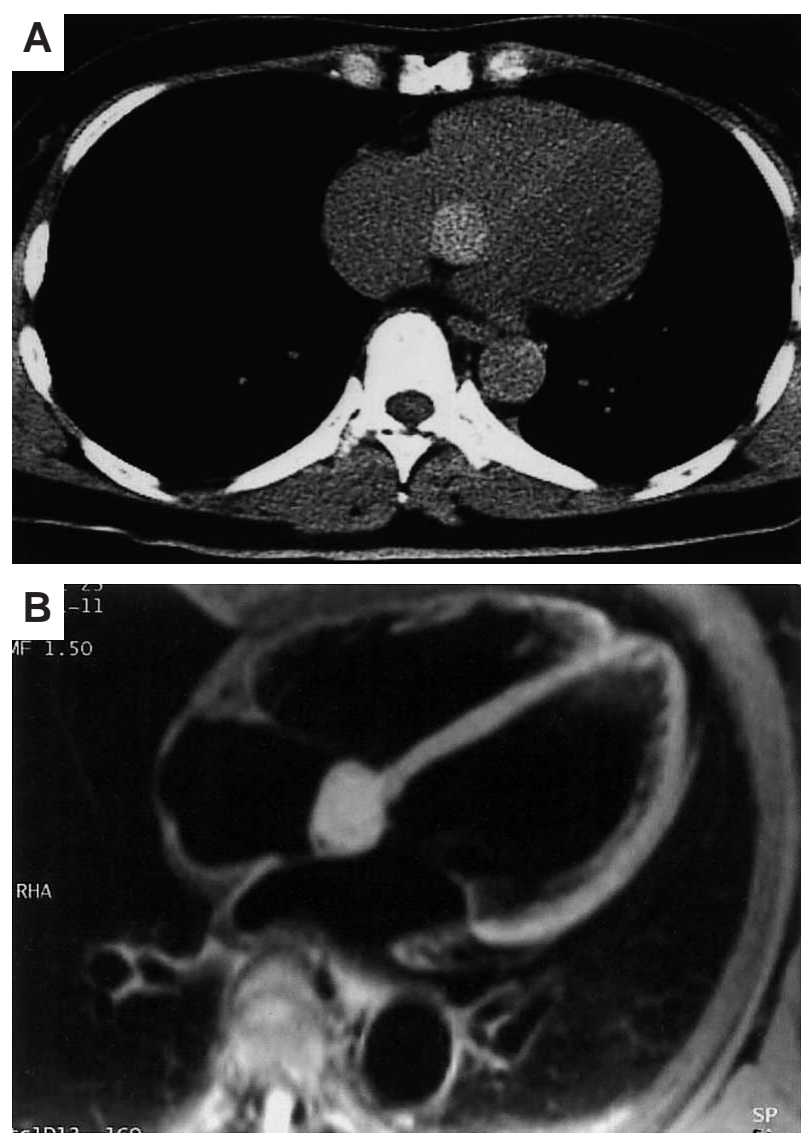

Fig 1. (A) Computed tomography scan and (B) magnetic resonance imaging showing intracardiac tumor with a broad connection to the interatrial septum. 


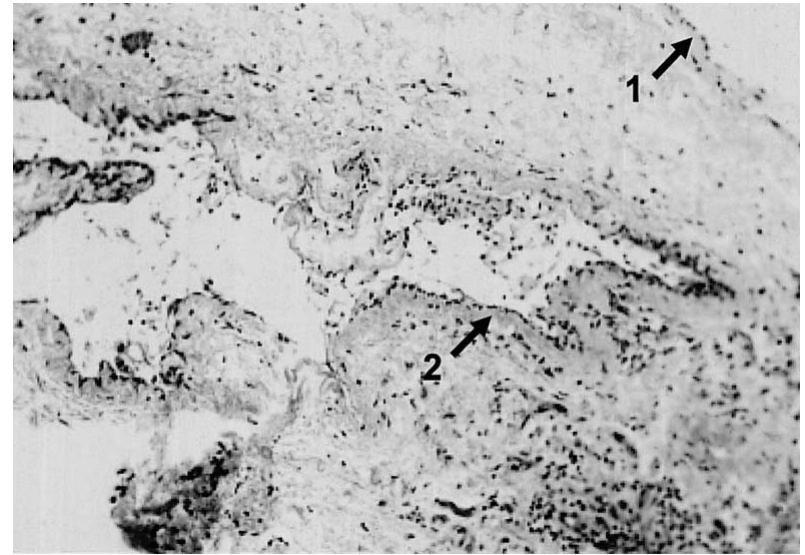

Fig 2. Photomicrograph (hematoxylin-eosin staining, $\times 400$ ) of the cyst wall showing the large cyst lining of the squamous epithelium (arrow 1), and within the fibrous wall of the large cyst, a smaller cyst lined by similar squamous epithelium (arrow 2 ) is shown.

atrioventricular node became a defect after the wall of the cyst was completely resected. It was closed directly with 6-0 monofilament sutures. Complete atrioventricular block persisted after surgery and a permanent pacemaker was inserted 11 days later.

Histopathological examination revealed that the cyst wall was composed of fibrous connective tissue covered by a layer of squamous epithelium with partial cornification. Within the fibrous tissue were smaller cysts lined by a similar epithelium (Fig 2). Immunohistochemical staining showed that the cells of the cyst expressed carcinoembryonic antigen and epithelial membrane antigen, suggesting that they were of endodermal origin.

The patient's recovery was uneventful and her symptoms completely disappeared. A 5-year follow-up has revealed no evidence of recurrence of the tumor.

\section{Discussion}

Cystic tumor of the atrioventricular nodal region was first described in 19115. It is a rare primary cardiac tumor located in the region of the atrioventricular node. According to previous reports ${ }^{1-3,6}$ it can cause variable degrees of heart blockage, which gradually evolve to complete heart blockage. Moreover, ventricular tachycardia or fibrillation sometimes occurs in patients with this tumor, $2,3,6$ Tumor size varies from $0.5 \mathrm{~mm}$ to $30 \mathrm{~mm}$, and there is no relationship between size and the occurrence of lethal arrhythmia. It is thus important to consider the possibility of cystic tumor of the atrioventricular nodal region in patients with electrocardiographic evidence of heart block limited to the atrioventricular node (ie, with a narrow QRS), particularly in women?

This tumor is thought to be of embryologic origin and not a true neoplasm because of its benign histological appearance and invariable location in the atrioventricular area. The atrioventricular nodal region is an area of embryologic fusion, suggesting that either mesothelium or nearby foregut inclusion could be incorporated in it. The immunohistochemical findings in this case, which corresponded with those of other recently reported cases, ${ }^{1,6-9}$ suggested that the tumor was an endodermal and not mesothelial remnant.

The cause of lethal arrhythmia in patients with cystic tumor of the atrioventricular nodal region is uncertain. Complete heart blockage or associated dysfunction of the sinus node might cause excessive distention of the ventricle and lead to ventricular fibrillation. However, this hypothesis does not explain why lethal arrhythmia often occurs just after the beginning of electronic pacing! 10

The first case of successful resection of this tumor was reported in 1992 by Balasundaram et al? In their case, the atrioventricular node tumor was only $0.5 \mathrm{~mm}$ in diameter and was an unexpected finding associated with an ostium secundum atrial septal defect. Paniagua et al reported the first case in which this type of tumor was detected preoperatively by echocardiography and MRI? Kaminishi et al recently reported the successful prevention of heart blockage by leaving the cyst wall attached to the base of the IAS? There was no sign of residual mass or recurrence 12 months after surgery. It is controversial whether the cyst should be resected completely from the base of the IAS. However, because one complication of this tumor is sudden death as a result of ventricular tachycardia or ventricular fibrillation, 2,3,6 we believe that complete resection is essential, even if subsequent pacemaker implant is required.

\section{References}

1. Bruke AP, Anderson PG, Virmani R, James TN, Herrera GA, Ceballos R. Tumor of the atrioventricular nodal region. Arch Pathol Lab Med 1990; 114: 1057-1062.

2. Nishida K, Kamijima G, Nagayama T. Mesothelioma of the atrioventricular node. Br Heart J 1985; 53: 468-470.

3. Travers H. Congenital polycystic tumor of the atrioventricular node: Possible familial occurrence and critical review of reported cases with special emphasis on histogenesis. Hum Pathol 1982; 13: $25-$ 35 .

4. Nojima Y, Ishibashi-Ueda H, Yamagishi M. Cystic tumor of the atrioventricular node. Heart 2003; 89: 122.

5. Armstrong H, Monckeberg JG. Herzblock, bedingt druch primaren Herztumor, bei einem 5 jahrigen Kinde. Dtsch Arch Klin Med 1911; 102: $144-166$.

6. Monma N, Sotodate R, Tashiro A, Segawa I. Origin of so-called mesothelioma of the atrioventricular node. Arch Pathol Lab Med 1991; 115: 1026-1029.

7. Balasundaram S, Halees SA, Duran C. Mesothelioma of the atrioventricular node: First successful follow-up after excision. Eur Heart $J$ 1992; 13: 718-719.

8. Paniagua JR, Sadaba JR, Davidson LA, Munsch CM. Cystic tumor of the atrioventricular nodal region: Report of a case successfully treated with surgery. Heart 2000; 83: E6.

9. Kaminishi Y, Watanabe Y, Nakata H, Shimokama T, Jikuya T. Cystic tumor of the atrioventricular nodal region. Jpn J Thorac Cardiovasc Surg 2002; 50: $37-39$.

10. James TN, Galakhov I. Fatal electrical instability of the heart associated with benign congenital polycystic tumor of the atrioventricular node. Circulation 1977; 56: 667-678. 